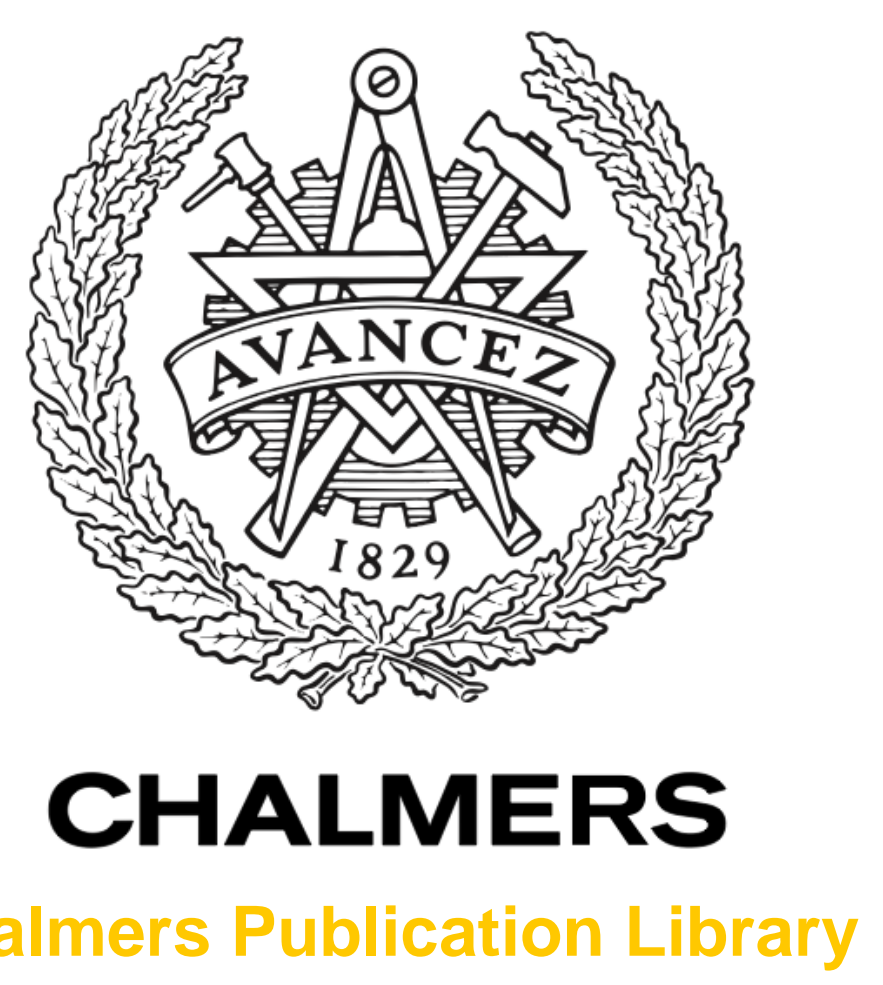

Chalmers Publication Library

\title{
Blind sub-Nyquist GNSS signal detection
}

This document has been downloaded from Chalmers Publication Library (CPL). It is the author's version of a work that was accepted for publication in:

41st IEEE International Conference on Acoustics, Speech and Signal Processing, ICASSP 2016, Shanghai, China, 20-25 March 2016 (ISSN: 1520-6149)

Citation for the published paper:

Daniel, O. ; Raasakka, J. ; Peltola, P. et al. (2016) "Blind sub-Nyquist GNSS signal

detection". 41st IEEE International Conference on Acoustics, Speech and Signal Processing,

ICASSP 2016, Shanghai, China, 20-25 March 2016 pp. 6575-6579.

http://dx.doi.org/10.1109/ICASSP.2016.7472944

Downloaded from: http://publications.lib.chalmers.se/publication/239094

Notice: Changes introduced as a result of publishing processes such as copy-editing and formatting may not be reflected in this document. For a definitive version of this work, please refer to the published source. Please note that access to the published version might require a subscription.

Chalmers Publication Library (CPL) offers the possibility of retrieving research publications produced at Chalmers University of Technology. It covers all types of publications: articles, dissertations, licentiate theses, masters theses, conference papers, reports etc. Since 2006 it is the official tool for Chalmers official publication statistics. To ensure that Chalmers research results are disseminated as widely as possible, an Open Access Policy has been adopted.

The CPL service is administrated and maintained by Chalmers Library. 


\title{
BLIND SUB-NYQUIST GNSS SIGNAL DETECTION
}

\author{
Ondrej Daniel*, Jussi Raasakka**, Pekka Peltola ${ }^{\ddagger}$,Markus Fröhle ${ }^{\S}$, \\ Alejandro Rivero-Rodriguez", Henk Wymeersch ${ }^{\S}$, and Jari Nurmi*
}

\author{
*Department of Electronics and Communications Engineering, Tampere University of Technology, Tampere, Finland \\ **Advanced Technology Europe, Honeywell International, Brno, Czech Republic \\ ${ }^{\ddagger}$ Nottingham Geospatial Institute, University of Nottingham, Nottingham, UK \\ ${ }^{\S}$ Department of Signals and Systems, Chalmers University of Technology, Gothenburg, Sweden \\ "Department of Mathematics, Tampere University of Technology, Tampere, Finland \\ ondrej.daniel@tut.fi,jussi.raasakka@honeywell.com, pekka.peltola@nottingham.ac.uk, \\ frohle@chalmers.se, alejandro.rivero@tut.fi, henkw@chalmers.se,jari.nurmi@tut.fi
}

\begin{abstract}
A satellite navigation receiver traditionally searches for positioning signals using an acquisition procedure. In situations, in which the required information is only a binary decision whether at least one positioning signal is present or absent, the procedure represents an unnecessarily complex solution. This paper presents a different approach for the binary detection problem with significantly reduced computational complexity. The approach is based on a novel decision metric which is utilized to design two binary detectors. The first detector operates under the theoretical assumption of additive white Gaussian noise and is evaluated by means of Receiver Operating Characteristics. The second one considers also additional interferences and is suitable to operate in a real environment. Its performance is verified using a signal captured by a receiver front-end.
\end{abstract}

Index Terms - Global Navigation Satellite Systems, signal detection, indoor/outdoor detection.

\section{INTRODUCTION}

We consider the problem of detecting a positioning signal used by Global Navigation Satellite Systems (GNSS). Traditionally, detection is performed by an acquisition procedure which returns rough estimates of signal parameters (time delay and Doppler frequency) for all discovered signals [1]. In contrast, we focus on a reduced problem which aims to detect only whether at least one positioning signal is available. The acquisition procedure can be exploited for this purpose as well. Also other detection procedures based on the cyclostationarity property of GNSS signals can be exploited [2]. However, these procedures exhibit high computational demands. Therefore, we propose a simpler detection approach requiring baseband samples captured with a low sampling frequency - significantly lower than the sampling frequencies typically used in GNSS receivers. This approach does not exploit knowledge of pseudorandom sequences. Both of these attributes lead to an implementation with low complexity.

The binary detection result is valuable in a variety of situations. The main use case scenario we are considering is evaluation whether the receiver is located indoors or outdoors. Different sensors provide different accuracies depending on the current operating environment

This work was supported by EU FP7 Marie Curie Initial Training Network MULTI-POS (Multi-technology Positioning Professionals) under grant no. 316528 and by the European Research Council under grant no. 258418 (COOPNET). (for example, GNSS receivers typically cannot produce a satisfactory position estimation indoors, but provide good position accuracy outdoors). By sensing the current operating environment, significant power savings can be achieved by turning off sensors that cannot provide accurate measurements in the current location. In [3], a lightweight indoor/outdoor detection method is presented, which utilizes a number of sensors typically found in a modern mobile phone. The authors claim that a detection accuracy of $88 \%$ is achieved using aggregate of three sensors; namely light, cellular, and magnetic field sensor. In [4], a semi-supervised learning approach is proposed for the same problem. It achieves $92.33 \%$ accuracy using seven different sensors.

The specific contributions of our work are as follows. First, we propose a novel decision metric calculated with low complexity. Second, we use the metric to design a binary detector operating in Additive White Gaussian Noise (AWGN) and analyze its performance by means of Receiver Operating Characteristics (ROC). Third, we consider additional interferences and we design another binary detector suitable for operation in a real environment. Finally, we utilize this detector for indoor/outdoor detection problem and verify its performance using a real signal.

\section{SIGNAL MODEL}

A receiver observes a passband GNSS signal at carrier frequency $f_{c}$. It is assumed that the signal is disturbed by AWGN as well as by other interferences, which are potentially non-stationary and non-Gaussian. The receiver shifts the signal into baseband and then filters it by a brickwall lowpass filter with a single-sided bandwidth $B$. It produces band-limited continuous-time signal $x(t)$ sampled at sampling frequency $f_{s}=1 / T_{s}=2 B$. The resulting complex discrete-time signal reads [5]

$$
x[n]=x\left(n T_{s}\right)=\sum_{i=0}^{S-1} x_{i}[n]+w[n]+z[n],
$$

where $S$ is the number of satellites from which the useful signals $x_{i}[n]$ are received, $w[n]$ represents independent samples of equivalent complex AWGN with Power Spectral Density (PSD) $2 N_{0}$ and variance $\sigma_{w}^{2}=4 N_{0} B$, and $z[n]$ models other disturbances.

Under the narrowband signal assumption, in which the effect of the Doppler frequency shift on signal bandwidth is neglected, the $i$ th useful signal is modeled as

$$
x_{i}[n]=a_{i} s_{i}\left(n T_{s}-\tau_{i}\right) e^{j 2 \pi f_{d_{i}} n T_{s}},
$$




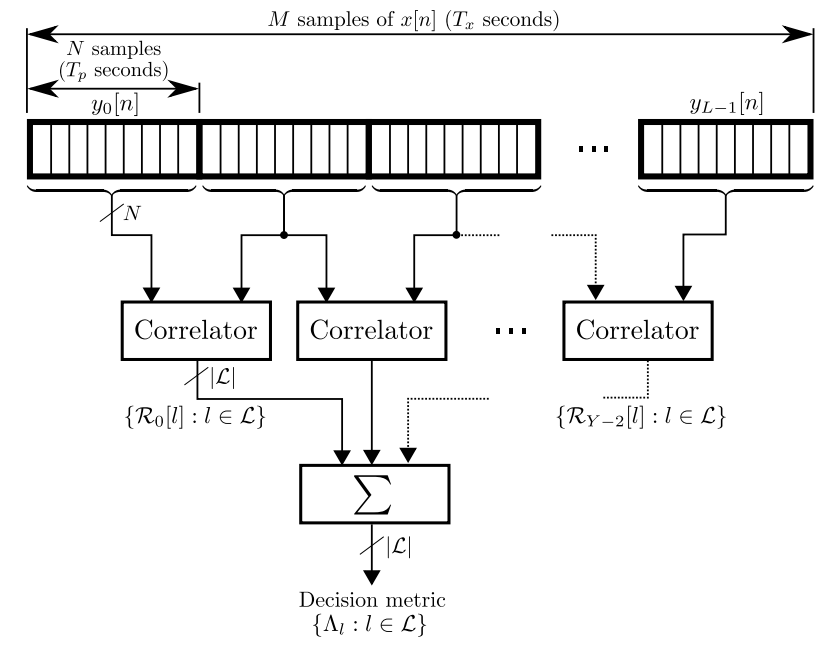

Fig. 1: Formulation of decision metric according to (5).

where $a_{i}$ is complex amplitude, $\tau_{i}$ is propagation delay, $f_{d_{i}}$ is the Doppler shift. Next, $s_{i}\left(n T_{s}\right)=s_{i}[n]$ are samples of $s_{i}(t)$, which is the bandlimited baseband equivalent of the transmitted signal. In general, the navigation signal $s_{i}(t)$ can encompass pseudorandom primary code optionally modulated by a variant of Binary Offset Carrier (BOC) modulation, pseudorandom secondary code, and navigation message [6]. One primary code period of $s_{i}(t)$ lasts $T_{p}$ seconds. Moreover, for each individual $i$ th useful signal it holds that its corresponding carrier-to-noise ratio equals $\left[C / N_{0}\right]_{i}=a_{i}^{2} /\left(2 N_{0}\right)$, since the power of the passband signal corresponding to the signal $x_{i}[n]$ equals $a_{i}^{2} / 2$ and one-sided PSD of passband noise is $N_{0}$ [5].

\section{PROPOSED METHOD}

The proposed method is a binary detector choosing between a noiseonly hypothesis $\mathcal{H}_{0}$ and a signal present hypothesis $\mathcal{H}_{1}$ :

$$
\begin{aligned}
& \mathcal{H}_{0}: x[n]=w[n]+z[n] \\
& \mathcal{H}_{1}: x[n]=\sum_{i=0}^{S-1} x_{i}[n]+w[n]+z[n] .
\end{aligned}
$$

A decision metric $\Lambda_{l}$ used to select one of these hypotheses is formed based on non-overlapping blocks of input signal $x[n]$ consisting of $M$ samples with total duration $T_{x}$ seconds. As illustrated in Fig. 1, the samples are further split into $L$ sub-blocks denoted $y_{i}[n]$, each with a length of $N$ samples or $T_{p}$ seconds. To form the decision metric, cross-correlations between adjacent sub-blocks are calculated first, leading to

$$
\mathcal{R}_{i}[l]=\sum_{n=0}^{N-1} y_{i}[n] y_{i+1}^{*}[n-l] \quad i=0,1, \ldots, L-2,
$$

where $l \in \mathcal{L} \subseteq \mathbb{Z}$ is a correlation lag. Second, these partial results are combined to finally produce the decision metric in the form of

$$
\Lambda_{l}=\sum_{i=0}^{L-2} \mathcal{R}_{i}[l]
$$

As it will be demonstrated further, the decision can be properly made on GNSS signals sampled with a very low sampling rate. In our case $f_{s}=50 \mathrm{kHz}$. We say that this is sub-Nyquist rate, since sampling rates required to properly capture GNSS signals are traditionally in the range of $\mathrm{MHz}$. Since signal model parameters are not required for calculation of the metric, we say it operates blindly. For the sake of clarity, in the analysis we focus primarily on L1 C/A signal used in Global Positioning System (GPS), and hence $T_{p}=1 \mathrm{~ms}$. We select the last design parameter $T_{x}=1 \mathrm{~s}$. This overall setting implies $N=50$ and $M=50 \cdot 10^{3}$ samples, and $Y=10^{3}$ blocks.

To make the final decision about the presence of navigation signals, we exploit the decision metric (5) differently depending on the considered signal disturbances. We distinguish between two cases: $A W G N$-only $(z[n]=0)$ and $A W G N$ plus interference $(z[n] \neq 0)$ disturbances.

\subsection{AWGN-only disturbance}

The stationarity of AWGN implies its constant stochastic characteristics. This allows designing the binary detector simply as a comparator of a predefined constant threshold $\gamma^{\prime}$ with the metric (5). The metric is evaluated only for zero lag $l=0$, since only then it can achieve significant correlation value. The detector decides $\mathcal{H}_{1}$ if

$$
\left|\Lambda_{0}\right|>\gamma^{\prime} \text {. }
$$

The probability density function of $x[n]$ under $\mathcal{H}_{0}$ depends only on noise variance $\sigma_{w}^{2}$, which is a constant value ${ }^{1}$. Therefore, threshold $\gamma^{\prime}$ maintains a constant probability of false alarm $P_{f a}$ [7]. In contrast, probability of detection $P_{d}$ is influenced by several factors, such as the signal bandwidth $B$, the number of available satellites $S$, carrier-to-noise ratios $\left[C / N_{0}\right]_{i}$ as well as randomness given by the presence of navigation messages. A consideration of all these effects complicates analytical derivation and thus we evaluate detector performance in terms of ROC curves which we obtain by means of Monte Carlo simulation. The ROC curves are depicted in Fig. 2. For example, for $P_{f a}=0.05$ and $C / N_{0}=40 \mathrm{~dB}-\mathrm{Hz}$, the detector achieves $P_{d}=0.89$ and $P_{d}=0.93$, in case of $S=5$ and $S=10$ satellites, respectively. This result demonstrates feasibility of our approach to detect significantly bandlimited GNSS signals.

\subsection{AWGN plus interference disturbance}

In practice, however, the GNSS signals are accompanied not only by AWGN but also by interfering signals [8]. In general, AWGN variance $\sigma_{w}^{2}$ varies, for example, with ambient temperature and antenna orientation. Also, the interfering signals may randomly change in time and in space. These changes lead to a continual variation of overall noise floor. To keep constant $P_{f a}$, the binary detector must adjust the decision making process to this variation. We roughly characterize the noise floor by evaluation of metric (5) for a nonzero lag, particularly for $l=1$. With a slight abuse of notation, we introduce $\Lambda_{0}[k]$ and $\Lambda_{1}[k]$ as useful and as noise representing signals. Inspired by a constant false alarm rate detector [9], we compute mean of $\left|\Lambda_{0}[k]\right|$ and square root of power estimate of $\Lambda_{1}[k]$, denoted as $u$ and $v$, respectively, both over sliding windows containing $K$ consecutive samples. The proposed detector, which is depicted in Fig. 3, decides $\mathcal{H}_{1}$ if

$$
\frac{u}{v}>\gamma
$$

where $\gamma$ is the decision threshold. Moreover, let $d[k] \in\{0,1\}$ be a signal indicating whether a GNSS signal is present (1) or absent (0). The detector produces an estimate of this signal, denoted as $\hat{d}[k]$.

\footnotetext{
${ }^{1}$ In practice, $\sigma_{w}^{2}$ is determined by the thermal noise, the receiver equivalent noise bandwidth, and the receiver noise figure.
} 


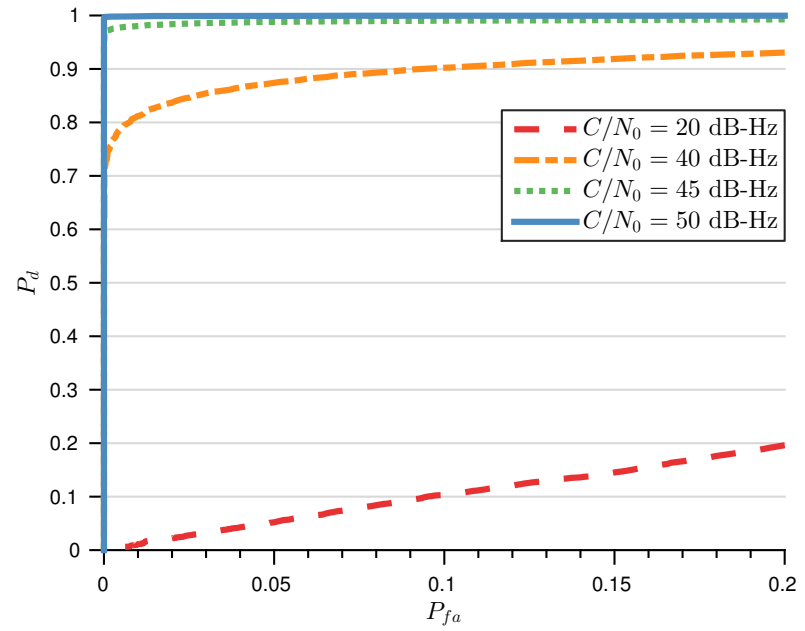

(a) Number of satellites $S=5$

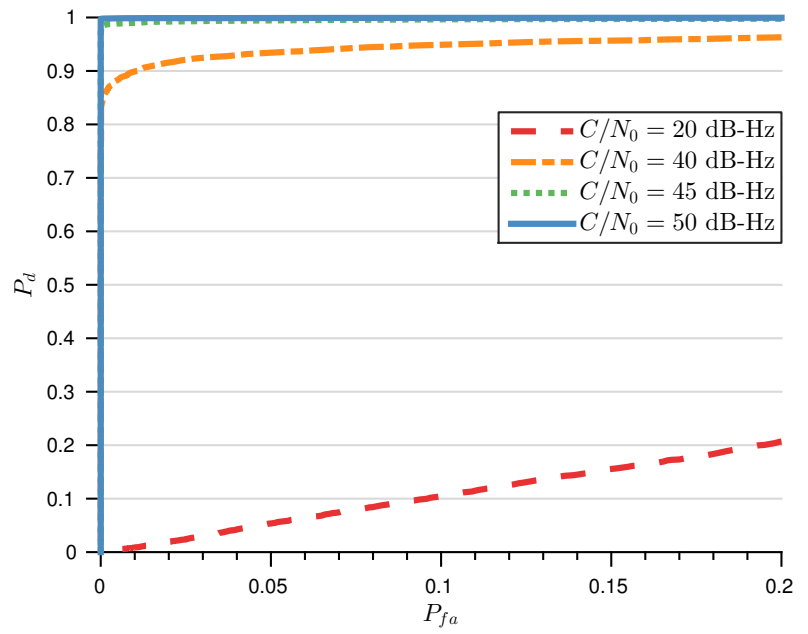

(b) Number of satellites $S=10$

Fig. 2: ROC curves for detector (6) operating under AWGN-only disturbance parametrized by number of available satellites $S$ and carrier-tonoise ratio $C / N_{0}$. To plot each curve, $10^{4}$ signal realizations are generated. Each signal consists of $S$ GPS L1 C/A signals summed together with AWGN according to (1) for $z[n]=0$. All of these $S$ signals have the same $\left[C / N_{0}\right]_{i}$ and their parameters are randomly generated from uniform distribution on the following intervals: phases $\angle\left(a_{i}\right)$ on $[0,2 \pi)$, Doppler shifts $f_{d_{i}}$ on $[-5,5] \mathrm{kHz}$, and time delays $\tau_{i}$ on $\left[0, T_{p}\right)$. Code numbers of used primary pseudorandom code sequences are generated randomly from uniform distribution on a discrete set $\{1, \ldots, 32\}$ and navigation message data bits are generated randomly as well. The signals are first generated with sampling rate $\tilde{f}_{s}=4 \mathrm{MHz}$ and then decimated (including proper lowpass filtering to avoid aliasing effect) to the desired sampling rate $f_{s}=50 \mathrm{kHz}$.

\section{PERFORMANCE RESULTS}

We evaluate performance of the proposed detector (7) on real measured data. We consider a use case scenario in which we estimate whether a receiver is located in an indoor or an outdoor environment based on the availability of the GPS signals.

First, it is necessary to properly set the detector constants $K$ and $\gamma$. For this purpose we have conducted a measurement campaign during which we have collected GPS signals using a capturing device based on SE4120L GNSS RF front-end [10], providing baseband samples at sampling rate $f_{s}^{\prime}=4.092 \mathrm{MHz}$. The duration of each record of the signals is roughly $90 \mathrm{~s}$ and during this time a user, equipped with the capturing device, slowly moves from outdoors to indoors. The time of transition, i.e. when the user goes

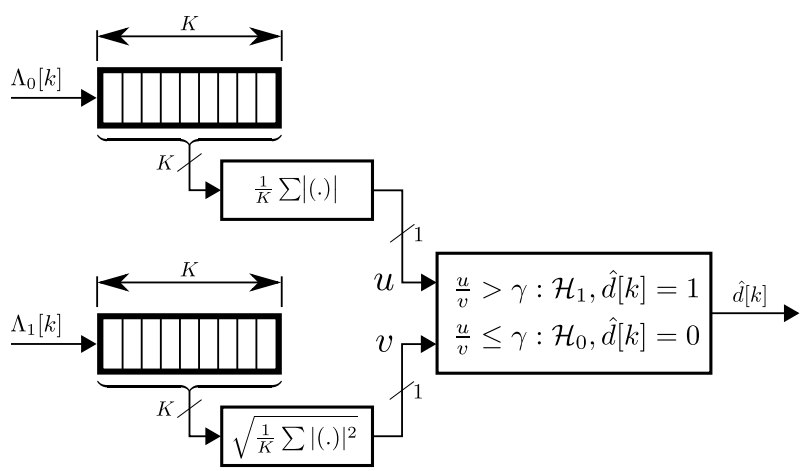

Fig. 3: Detector proposed for AWGN plus interference scenario. It operates on decision metric $\Lambda_{l}, l \in\{0,1\}$ calculated using (5). through the door, is recorded as well. In total, we have captured 22 indoor/outdoor transitions, each of them at different, randomly selected, places $(9 \times$ a residential place, $6 \times$ University of Tampere, $7 \times$ Tampere University of Technology). The captured signals are filtered, interpolated, and decimated to a low sampling rate $f_{s}=$ $50 \mathrm{kHz}$ as illustrated in Fig. 4. Next, the metrics $\Lambda_{0}[k]$ and $\Lambda_{1}[k]$ are computed for each of these resampled signals using (5). An example of the metrics for one of the collected signals is depicted in Fig. 6. The true transition signal $d[k]$ indicates that the user moved indoor at time $t=41 \mathrm{~s}$. Using the 22 sets of signals $\Lambda_{0}[k], \Lambda_{1}[k]$, and $d[k]$, the detector parameters $K$ and $\gamma$ are optimized to achieve the best agreement between all pairs of signals $\hat{d}[k]$ and $d[k]$. From this supervised learning process we get $K=6$ and $\gamma=3.4$.

Second, these parameters have been applied for a performance evaluation of the proposed method (7) on a test signal, which was recorded on the campus of Tampere University of Technology on different day and time than the training signals to ensure different conditions. The duration of the record is $600 \mathrm{~s}$ and it includes several indoor/outdoor transitions. The final result is depicted in Fig. 5. In this particular case, the detector is successful during $92.26 \%$ of the time.

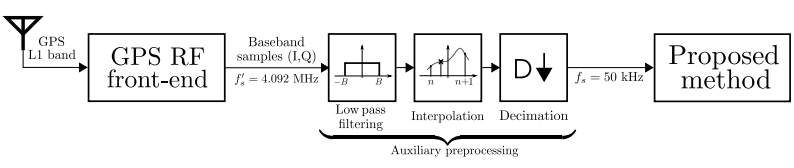

Fig. 4: Real signal capturing and preprocessing. The sampling rate is reduced from $f_{s}^{\prime}=4.092 \mathrm{MHz}$ to $f_{s}=50 \mathrm{kHz}$ using lowpass filtering, interpolation and decimation. The interpolation is required due to a non-integer decimation factor. 


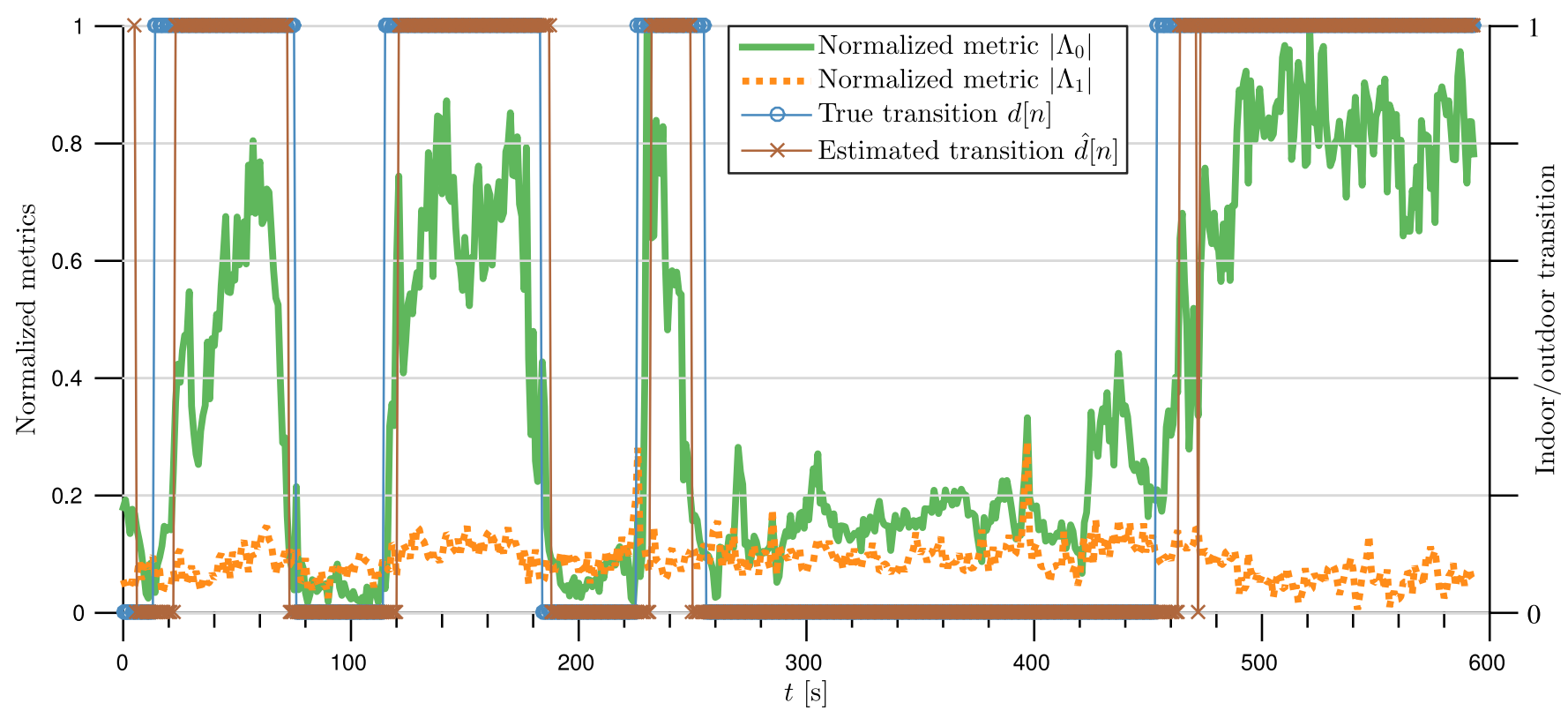

Fig. 5: Evaluation of proposed detector (7) in a real scenario. In this case, the detector returns a correct decision during $92.26 \%$ of the time.

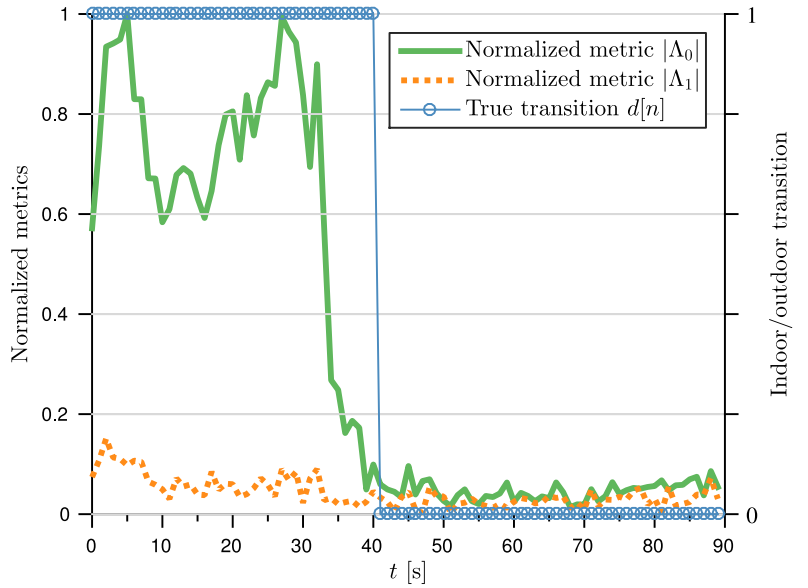

Fig. 6: An example of post processing of a signal recorded during the measurement campaign. The transition happens at $t=41 \mathrm{~s}$.

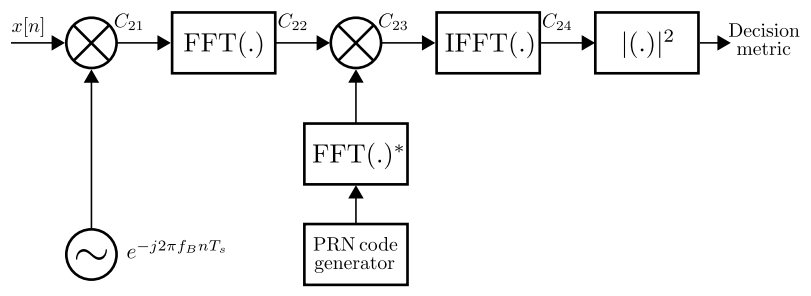

Fig. 7: Acquisition unit using parallel code space search approach. The variables $C_{21}, C_{22}, C_{23}$, and $C_{24}$ denote number of complex multiplications calculated by corresponding blocks.

\section{COMPLEXITY EVALUATION}

The complexity of the proposed detector (7) is evaluated in terms of the number of complex multiplications. It is compared with the com- plexity of a traditional acquisition unit using a parallel code space search approach [11]. The unit is depicted in Fig. 7.

To calculate $\Lambda_{l}$ for $l \in\{0,1\}$, the proposed method requires $C_{1}=2 N Y=10^{5}$ multiplications.

For the complexity evaluation we use the following assumptions. The acquisition unit performs coherent integration over $T_{E}=1 \mathrm{~ms}$ of the positioning signal sampled with rate $f_{s}^{\prime}=4.092 \mathrm{MHz}$. Hence it operates on $U=T_{E} f_{s}^{\prime}=4092$ samples. The Doppler frequency search range is $[-5,5] \mathrm{kHz}$ with step $500 \mathrm{~Hz}$ yielding $V=20$ frequency bins $f_{B}$. The unit needs to search for $W=4$ satellites on average in order to find a visible satellite. The acquisition procedure is performed once every $T_{X}=1 \mathrm{~s}$. Therefore, the unit requires $C_{2}=C_{21}+C_{22}+C_{23}+C_{24}=U V+V(U / 2) \log _{2}(U)+U V W+$ $V W(U / 2) \log _{2}(U)=2.864 \cdot 10^{6}$ complex multiplications.

Under these assumptions, our method requires $C_{1} / C_{2} \approx 29$ times less complex multiplications than the acquisition unit.

\section{CONCLUSION}

We have considered the problem of binary GNSS signal detection for which we have introduced a decision metric computed with low complexity. Using the metric we have designed two binary detectors. The first detector, operating in AWGN channel, has been used to demonstrate the feasibility of performing GNSS signal detection without knowledge of underlying signal parameters and on input samples acquired with very low sampling rate. The second one has been designed to operate in a real environment. We have exploited this detector for the purpose of indoor/outdoor detection and we have evaluated its proper functionality using a real signal. The detector has provided the correct decision during $92.26 \%$ of the time. We have found that the complexity of our proposed detector is a factor of 29 times lower compared to the complexity of a traditional GNSS acquisition unit utilizing parallel code space search approach. 


\section{REFERENCES}

[1] Bradford W. Parkinson and James J. Spilker, Global Positioning System: Theory and Applications, Volume 1, vol. 1, American Institute of Aeronautics \& Astronautics, 1996.

[2] E. S. Lohan, J. Lundn, G. Seco-Granados, J. A. Lopez-Salcedo, and V. Koivunen, "Cyclic Frequencies of BOC-Modulated GNSS Signals and Their Potential Within a Cognitive Positioning Framework," Navigation, vol. 61, no. 2, pp. 95-114, 2014.

[3] Pengfei Zhou, Yuanqing Zheng, Zhenjiang Li, Mo Li, and Guobin Shen, "IODetector: A Generic Service for Indoor Outdoor Detection," in Proceedings of the 10th ACM Conference on Embedded Network Sensor Systems, New York, NY, USA, 2012, SenSys '12, pp. 113-126, ACM.

[4] Valentin Radu, Panagiota Katsikouli, Rik Sarkar, and Mahesh K. Marina, "A Semi-supervised Learning Approach for Robust Indoor-outdoor Detection with Smartphones," in Proceedings of the 12th ACM Conference on Embedded Network Sensor Systems, New York, NY, USA, 2014, SenSys '14, pp. 280-294, ACM.

[5] Gonzalo Seco-Granados, Jose Lopez-Salcedo, David JimenezBanos, and Gustavo Lopez-Risueno, "Challenges in Indoor Global Navigation Satellite Systems: Unveiling its core features in signal processing," IEEE Signal Processing Magazine, vol. 29, no. 2, pp. 108-131, Mar. 2012.

[6] Jaume Sanz Subirana, José Miguel Juan Zornoza, and Hernández-Pajares Manuel, GNSS Data Processing, Vol. I: Fundamentals and Algorithms, ESA Communications, 2013.

[7] Steven M. Kay, Fundamentals of Statistical Signal Processing, Volume II: Detection Theory, Prentice-Hall PTR, New Jersey, 1998.

[8] Ren Jr. Landry and Alain Renard, "Analysis of potential interference sources and assessment of present solutions for GPS/GNSS receivers," 4th Saint-Petersburg on INS, pp. 1-13, 1997.

[9] Mark A. Richards, Fundamentals of Radar Signal Processing, Second Edition, McGraw-Hill, 2014.

[10] SE4120L GNSS Receiver IC, SiGe Semiconductor, 2009.

[11] James Bao-Yen Tsui, Fundamentals of Global Positioning System Receivers: A Software Approach, John Wiley \& Sons, 2005. 